\title{
Corpus
}

\section{Intertexte générique et interprétation des actes de parole dans un corpus d'émissions de plateaux télévisées}

\section{Nicolas Desquinabo}

\section{CpenEdition}

Journals

Édition électronique

URL : http://journals.openedition.org/corpus/1192

DOI : $10.4000 /$ corpus. 1192

ISSN : 1765-3126

Éditeur

Bases; corpus et langage - UMR 6039

Édition imprimée

Date de publication : 1 décembre 2007

Pagination : 127-152

ISSN : 1638-9808

\section{Référence électronique}

Nicolas Desquinabo, «Intertexte générique et interprétation des actes de parole dans un corpus d'émissions de plateaux télévisées », Corpus [En ligne], 6 | 2007, mis en ligne le 02 juillet 2008, consulté le 08 septembre 2020. URL : http://journals.openedition.org/corpus/1192 ; DOI : https:// doi.org/10.4000/corpus. 1192 


\title{
Intertexte générique et interprétation des actes de parole dans un corpus d'émissions de plateaux télévisées
}

\author{
Nicolas DESQUINABO \\ Université de la Sorbonne Nouvelle - Paris 3
}

La quantité de dimensions contextuelles potentiellement utilisées dans l'interprétation des discours pose un problème central à toute tentative d'explicitation des parcours interprétatifs effectivement utilisés par un lecteur, interlocuteur ou auditeur (Kleiber et al., 2006). A partir d'un questionnement sur l'interprétation des actes de parole échangés à l'occasion d'interactions télévisées, nous avons mis à l'épreuve plusieurs hypothèses sur l'articulation des différentes dimensions contextuelles et textuelles dans l'interprétation des discours.

Tout d'abord, nous soutiendrons qu'interpréter un texte écrit ou oral consiste au moins en partie à segmenter ce texte en différentes actes, à leur attribuer une visée illocutoire manifeste (demande d'information, compliment, etc.) et à relier entre eux certains de ces actes (information justifiant un désaccord, information répondant à une demande, etc.). Selon nous, ces processus interprétatifs se déroulent généralement à partir d'hypothèses contextuelles sur le genre de discours pratiqué par le ou les énonciateur(s) du texte. Nous défendrons dans cet article l'idée que les genres de discours associent des hiérarchies d'actions typiques à des « intertextes génériques » et à une configuration d' "indices génériques », l'ensemble étant situé dans des domaines et champs génériques (Rastier, 2001). A l'aide d'indices pluri-sémiotiques, une situation d'action est identifiée puis référée à un type de pratique sociale dont elle semble être une occurrence (Rastier, 1998 ; Filliettaz, 2006). Cette indexation mobilise un intertexte générique qui oriente les processus interprétatifs, en particulier s'agissant de l'attribution des valeurs illocutoires et interactives probables des actes de 
NiCOLAS DESQUINABO

parole. La récurrence de ces valeurs constitue en retour des indices potentiels du genre de discours en cours.

Nous présenterons dans cet article deux mises à l'épreuve de ces hypothèses. Nous décrirons tout d'abord notre analyse "globale " d'un corpus de vingt-quatre interactions télévisées et de leur péritexte. Cette analyse nous a permis d'observer des correspondances entre différentes configurations lexicales et morphosyntaxiques et quatre schémas d'actes de parole mis en évidence dans une précédente recherche (Desquinabo, 2005). Dans un second temps, nous soulignerons certaines incidences interprétatives plus «locales » des prescriptions génériques, à l'aide d'une analyse des parcours probables de nos interprétations au palier de la période ${ }^{1}$.

Nous conclurons sur les principales limites qui affectent nos hypothèses et résultats : difficultés économiques liées à l'analyse des actes de parole sur de larges corpus et problèmes posés par le repérage des changements d'activités discursives. Pour finir, nous ouvrirons une réflexion sur les interactions multiples entre contexte générique et plans du discours (non seulement actes de parole, mais aussi progressions thématiques, prises en charge énonciatives, etc.).

\section{Cadre théorique «psycho-socio-pragmatique » des genres de discours}

Notre point de vue théorique se situe au point de convergence de l'ethnographie de la communication de Gumperz (1989) et Hymes (1980), de la psycholinguistique (Coirier, Gaonac'h \& Passerault, 1996) et de la tradition rhétorique / herméneutique (Rastier, 2001). Cette conception soutient la centralité des normes sociales et notamment des genres de discours dans la production et l'interprétation des discours (Rastier, 1994: 68 ; Bronckart, 1997: 137 ; Chabrol \& Bromberg, 1999: 296). Nous inspirant de François Rastier (2001 : 230-231) et Marcel Burger (2001), nous situons la catégorie des genres de discours

1. Nous utilisons le terme «période » au sens de Rastier (1994: $64 ; 2001$ : 264). Ce palier, situé à l'écrit entre le syntagme et la section, est particulièrement délicat à délimiter dans les textes interlocutifs oraux. 
Intertexte générique et interprétation des actes de parole

à l'intérieur de domaines d'activités (littéraire, judiciaire, télévisuel) scindés en champs génériques homogènes du point de vue de l'organisation énonciative et/ou sémiotique (plateau, reportage, fiction et spectacle pour la télévision). Ainsi situés, les genres sont des catégories discursives associant des hiérarchies de "visées énonciatives » (Charaudeau, 1995 : 153) à des schématisations discursives normées, disponibles en mémoire à long terme. Dans les interactions, ces schématisations concernent les différents plans du discours, dont les tours de parole, actes de paroles, lexiques et parcours interprétatifs privilégiés. Ces schémas sont plus ou moins normés selon les genres et plans discursifs et peuvent être partiellement négociés au cours des interactions successives (Bange, 1989). En outre, les genres et leurs normes sont partagés par des communautés socio-discursives caractérisées par leur "expérience » discursive commune. Cette familiarité aux mêmes discours permet de reconnaître les genres de manière approximativement identique, à l'aide de configurations d'indices paratextuels et/ou textuels récurrents. Enfin, chaque " texte » limité spatialement ou temporellement (rencontre, émission, ouvrage, etc.) peut donner lieu à l'utilisation d'un ou plusieurs genres, éventuellement situés dans différents discours.

\subsection{Paliers et types d'action discursive}

Notre cadre théorique général présenté, il nous faut préciser nos hypothèses sur les rapports entre paliers textuels et paliers de l'action ${ }^{2}$. En premier lieu, rappelons notre hypothèse selon laquelle interpréter un texte écrit ou oral consiste au moins en partie à segmenter ce texte en différents actes, à leur attribuer une visée illocutoire manifeste (demande d'information, compliment, ordre, etc.) et à relier entre eux certains de ces actes (information justifiant un accord ou répondant à une demande, etc.). Notre définition du concept d' ' acte de parole » est en partie traditionnelle: il s'agit de l'unité communicationnelle minimum ayant un contenu (généralement) «proposi-

2. Ces hypothèses s’inspirent en particulier des travaux de Bange (1989) et de Filliettaz (2001) 


\section{NiCOLAS DESQUiNABO}

tionnel », une visée « illocutoire » (en situation) et un (éventuel) effet « perlocutoire ». Les actes de parole sont utilisés dans des unités communicationnelles supérieures (Bange, 1989) dont celle de l' «interaction». Cette unité contient généralement différentes "périodes" dans lesquelles se déploient des "échanges", eux-mêmes composés d'actes de parole ${ }^{3}$. L'ensemble de ces unités se typifient par leur pratique sociohistorique. En particulier, la pratique récurrente d'interactions à visées énonciatives équivalentes conduit à la stabilisation progressive de genres d'activités discursives.

Afin d'attribuer une valeur illocutoire aux actes de parole de notre corpus, nous avons utilisé la classification mise en place par Chabrol et Bromberg (1999) ${ }^{4}$. Ces auteurs distinguent environ cinquante types d'actes de parole classés dans cinq sphères (information, évaluation, interaction, action, métacommunication). La segmentation du texte en actes correspond généralement au découpage en propositions " complètes » ou « implicitées » (oui, d'accord, etc). Tous les actes de chaque sphère peuvent être donnés, demandés et acceptés ou refusés : ce sont les modalités «interlocutrices ». "Accepter d'informer » c'est le faire après qu'on nous l'ait demandé, alors que « donner une information » signifie qu'elle ne nous a pas été demandée ${ }^{6}$. Suivant Roulet (2001), notre modèle distingue également plusieurs types de relations entre

3. Par exemple, dans Hutchby \& Woofit (1998: 149-151), les multiples " demandes de reconnaître " adressées à la victime par l'avocat de la défense forment une "période" visant à construire " an image of the victim as a woman with loose morals » afin de gagner le procès qui est une succession de "rencontres » dans lesquelles se succèdent et s'enchâssent différentes interactions indexées à des "genres de discours » (rappel des faits, interrogatoires, plaidoiries, etc.).

4. Voir Desquinabo (2005: 88-91) pour une présentation détaillée de cette classification (provisoire) des types d'actes de parole.

5. Nous n'avons pas approfondi ici l'étude des processus de segmentation. Pour plus de détails sur ce point, voir la discussion du critère du " passage en mémoire discursive » par Roulet (2001: 65).

6. Par rapport aux modèles genevois (Roulet, 2001) ou conversationnaliste (Schegloff, 1984), nous distinguons les modalités «donner» et « demander » à l’intérieur de la fonction initiative. 
actes de parole ${ }^{7}$ ou « valeurs interactives » (argument et contreargument, explicitation, succession, préalable, topicalisation et commentaire).

Par rapport aux classifications des actes de langage de Searle (1982) ou de Vernant (1997), la classification de Chabrol \& Bromberg (1999) détaille en particulier les actes dits d' « interaction ». Ces actes (louer, blâmer, accuser, etc.) sont à la fois distingués de manière " ordinaire » et étudiés au moins depuis les travaux de la rhétorique antique (Rastier, 2001 : 135). Cette sphère correspond en partie aux "expressifs » de Searle $(1982)^{8}$. Cette taxinomie ne prétend évidemment pas à l'universalité (Bouchard, 1988: 105) mais tente de concilier l'instabilité des classifications ordinaires et l'explicitation des catégories scientifiques (Kerbrat-Orecchioni, 2005 : 81-82).

\subsection{Hypothèses sur les processus d'interprétation des actes de parole}

Nous proposons ici une modélisation du processus d'interprétation des actes de parole qui articule deux processus interdépendants aux paliers du texte et de la période: l'indexation du texte interlocutif à un intertexte générique et l'interprétation des actes de parole au «fil» du texte. Ces processus sont interdépendants car l'interprétation des actes de parole nécessite généralement l'indexation du texte en cours à un contexte générique, et cette indexation repose en partie sur l'identification d'actes récurrents dans un genre d'activité

7. A ce plan des relations « interactives » entre constituants du discours, notre modèle d'analyse diffère du modèle genevois dans l'application du principe de récursivité : nous n’avons pas analysé les relations interactives entre actes de participants différents (au niveau de l'échange). Pour nous, un échange peut certes être utilisé comme argument par un locuteur A, mais l'intervention du locuteur B ne change pas pour autant de valeur illocutoire et interactive pour ce dernier.

8. Moins étudiés que d'autres, les types d'actes «interactifs » restent à préciser compte tenu de la complexité des liens entre visées « relationnelles », « identitaires » et « émotionnelles » (KerbratOrecchioni, $2000: 52$ ) et de la question du palier de ces actes (souvent des macro-actes utilisant différents actes minimaux, comme par exemple l' " histoire drôle »). 
NiCOLAS DESQUiNABo

discursive donné. Selon nos hypothèses, l'interprétation d'une interaction télévisée comme de tout autre texte suppose :

- l'activation d'hypothèses praxéologiques sur les actions " globales » en cours,

- à partir de la reconnaissance de configurations d'indices plurisémiotiques,

- qui sélectionnent un «intertexte générique » utilisé pour orienter productions et parcours interprétatifs.

Parmi les parcours interprétatifs orientés par la sélection d'un intertexte générique figure celui de l'interprétation des valeurs interactives et illocutoires des actes de parole. Ce processus combine en particulier des attentes sur les actes plus ou moins probables à des indices lexicaux et morphosyntaxiques des différents actes de parole.

La notion d' ' intertexte générique » est utilisée ici dans un sens assez spécifique. Elle correspond en partie à l' " architextualité » de Gérard Genette (1979) dans la mesure où il ne s'agit pas d'une relation entre deux occurrences de textes, mais entre un texte et une catégorie générique de texte. Dans notre modélisation, un regroupement générique de textes est avant tout réalisé à partir de l'identification de hiérarchies d'actions de même type (mettre en valeur un invité en narrant certaines étapes de sa vie, convaincre les téléspectateurs en disqualifiant un interlocuteur, etc.) dans un champ générique donné (plateau, reportage, fiction, etc.). Nous faisons également l'hypothèse que l'intertexte générique est essentiellement mémorisé sous la forme de schémas d'actes de parole, thèmes, textures énonciatives ou vocabulaires plus ou moins stabilisés et plus ou moins « scriptés ».

Nous utilisons la notion d'indice générique dans le sens où, comme les actes de parole, les genres discursifs n'ont pas de " marqueurs » systématiquement associés à leur actualisation. Ces indices peuvent être pluri-sémiotiques et être situés dans le paratexte et/ou le texte (Rastier, 1994 : 167 ; Gumperz, 1989 : 29-43 ; Filliettaz, 2004 : 50). Les éventuels indices paratextuels du genre d'activité discursive à venir sont ainsi complétés par des indices prélevés dans le texte qui peuvent confirmer ou réorienter l'attribution générique initiale. Ces indices sont 
Intertexte générique et interprétation des actes de parole

graduels : ils sont reconnus comme "écart à la normale » (Gumperz, 1989 : 72) et peuvent donc être identifiés en raison de leur sous ou sur-emploi par rapport à la norme d'un corpus de référence. Compte tenu de ce cadre théorique, un tel corpus de référence doit être composé d'un échantillon de textes d'un même champ générique pratiqués en synchronie dans une communauté socio-discursive donnée ${ }^{9}$. Au delà des types d'indices étudiés dans la présente recherche (lexèmes, syntagmes, catégories morphosyntaxiques, actes de parole, nombre et orientation des participants, mobilier, taille de l'espace), le contexte générique peut être proposé ou reconnu à l'aide d'indices posturaux, mimico-gestuels, plastiques, musicaux ou thématiques.

Ainsi, selon notre modèle, les indications " linguistiques» des actes de parole (Searle, 1982) ou le "principe d'interprétation séquentielle » (Schegloff \& Sacks, 1973 : 295-299) ne suffisent pas à rendre compte de l'actualisation des actes de parole en contexte. Ne précisant pas les modalités d'interprétation des actes initiatifs (KerbratOrecchioni, $2005: 58-60$ ), l'interprétation séquentielle ne permet pas d'identifier les refus de répondre, réponses «à côté ", changements de genre, modifications thématiques ou réponses "retardées». En revanche, la combinaison des indications linguistiques aux instructions globales et locales de l'intertexte générique permet d'expliquer plus précisément l’interprétation des actes de parole.

\section{Première mise à l'épreuve de notre modélisation}

Cette première mise à l'épreuve vise à tester si d'éventuelles correspondances pourraient être observées entre différentes configurations lexico-syntaxiques textuelles et péritextuelles et quatre schémas d'actes de parole mis en évidence dans une précédente recherche (Desquinabo, 2005).

9. Nous n’abordons donc pas les problèmes d’instabilité générique posés par certains textes ré-édités et lus dans des contextes socio-historiques très variés (Adam \& Heidmann, 2004). 
NiCOLAS DESQUINABO

\subsection{Corpus}

Lors de cette étude, nous avons confronté nos hypothèses sur quatre genres interlocutifs supposés (controverse, portrait, polémique et jugement ${ }^{10}$ ) à l'analyse pragmadiscursive, lexicale et morphosyntaxique d'un corpus de 24 interactions de trois minutes $^{11}$, 6 par genre. Nous avons sélectionné ce corpus à partir d'hypothèses sur le champ générique des plateaux télévisés (vs reportages, fictions et spectacles) au sein duquel nous distinguons douze genres principaux ${ }^{12}$. L'analyse de ce corpus (codé « texte de l'interaction ») a été complétée par une analyse des éléments du "décor» de ces textes (codé " péritexte de l'interaction ») et du corpus des présentations ${ }^{13}$ des 16 émissions ${ }^{14}$ dans lesquelles ont été sélectionnées ces interactions (codé « péritexte de l'émission ») ${ }^{15}$.

\subsection{Indices génériques pragmadiscursifs}

A l'occasion d'une précédente recherche (Desquinabo, 2005), nous avons systématiquement noté les tours de parole (nombre, durée, distribution) et actes de parole (selon la grille de Chabrol

10. Dans le genre controverse, il s'agit essentiellement d'argumenter à partir d'évaluations pour ou contre une règle ou action commune sans mettre en cause personnellement son adversaire (sinon la polémique est déclenchée). La visée essentielle du genre jugement est de mettre en cause ou louer son interlocuteur à partir d'évaluations diverses alors que le genre portrait interdit la mise en cause de l'interlocuteur et privilégie la narration d'étapes de sa vie et l'expression de ses préférences.

11. Pour des raisons économiques, nous avons limité l'analyse aux trois premières minutes des 24 interactions.

12. Voir Desquinabo (2005) pour plus de détails sur ces douze genres « interlocutifs ».

13. Les présentations sont les monologues généralement énoncés par les animateurs en début d'émission et introduisant les genres, thèmes et/ou invités à venir.

14. Nous avons sélectionné les 24 interactions à l'intérieur de 16 occurrences d'émissions : 2 interactions dans 8 émissions et 1 interaction dans les 8 autres émissions.

15. Sur la distinction de ces corpus, voir Malrieu \& Rastier (2001: 553). Selon ces auteurs, les variables péritextuelles sont des variables contextuelles «globales» qui déterminent les variables textuelles «locales». Suivant cette conception, nous avons donc codé distinctivement et mis en relation ces deux types de corpus. 
Intertexte générique et interprétation des actes de parole

\& Bromberg, 1999). Nous avons alors soumis nos analyses à deux ensembles de traitements statistiques différents: multidimensionnels et descriptifs ${ }^{16}$. L'analyse en composantes principales (ACP) et la classification hiérarchique ${ }^{17}$ des variables caractérisant les animateurs et les invités ont permis de distinguer trois ensembles de comportements discursifs assez homogènes pour les invités (controverse, portrait et polémiquejugement: cf. graphique $\left.\mathrm{n}^{\circ} 2\right)$ et trois ensembles moins homogènes pour les animateurs (controverse, polémique et portrait-jugement). Les comparaisons des moyennes et coefficients de variation des différentes variables ont permis ensuite de préciser les schémas de tours et actes de parole typiques des animateurs et invités dans les quatre genres étudiés (tableau $\mathrm{n}^{\circ} 1$ ). Selon notre modèle, ces schémas constituent à la fois des instructions sur les productions et les interprétations des actes de parole et à la fois des indices utilisés par les téléspectateurs pour reconnaître le genre d'une interaction donnée.

\section{Controverse}

Animateur de débat $=$ demande d'évaluer $(60 \%)$ et régule un peu (18\%) ou "animateur-participant $=$ informe $(20 \%)$, demande d'évaluer (40\%) et évalue (15\%)

Invités = évaluent (21\%) et argumentent à partir d'évaluations (40\%) dans des tours de parole très longs (33s)

16. L’analyse descriptive et inférentielle précise les «spécificités » (Lebart \& Salem, 1994 : 171-177) et homogénéités (coefficients de variations) des types préalablement construits à partir d'hypothèses " externes » mais ne permet pas de tester «la répartition des textes retenus sous les rubriques choisies" (Habert, Nazarenko \& Salem, 1997: 28). L'utilisation de ces deux démarches permet en outre de tester la fiabilité des résultats obtenus par chacune d'entre elles séparément.

17. Ces analyses ont été réalisées à l'aide du logiciel Nestor édité par l'association PADI. 
NiCOLAS DESQUiNABO

\begin{tabular}{|c|}
\hline Portrait \\
\hline $\begin{array}{l}\text { Animateur = demande de narrer }(25 \%) \text {, narre }(15 \%) \text { et } \\
\text { complimente }(7 \%) \text { dans des tours assez courts }(7 \mathrm{~s}) \\
\text { Invités = narrent les événements de leur vie }(51 \%) \text {, expriment } \\
\text { leurs « goûts » (20\%) dans des tours courts (9s) }\end{array}$ \\
\hline Jugement \\
\hline $\begin{array}{l}\text { Animateur = blâme et demande des justifications (50\%) dans } \\
\text { des tours très courts (5s) } \\
\text { Invités = démentent, se justifient et blâment (68\%), évaluent } \\
\text { peu }(15 \%) \text { dans des tours très courts (5s) fréquemment } \\
\text { interrompus }(7 / \mathrm{mn})\end{array}$ \\
\hline Polémique \\
\hline $\begin{array}{l}\text { Animateur de débat = demande d'évaluer (42\% ) et régule un } \\
\text { peu (20\%) ou «animateur-régulateur » : régule (à 95\%) avec } \\
\text { un capital verbal très faible }(10 \%) \\
\text { Invités = démentent et blâment }(62 \%) \text { et évaluent }(25 \%) \text { en } \\
\text { s'interrompant fréquemment }(6 / \mathrm{mn})\end{array}$ \\
\hline
\end{tabular}

Tableau n²1. Schémas génériques d'actes et de tours de parole des animateurs et invités

Parmi l'ensemble des autres indices génériques potentiels des interactions de notre corpus, nous avons alors recherché quels pouvaient être les indices lexicaux et morphosyntaxiques des genres portrait, controverse, polémique et jugement. Afin de mettre à jour ces éventuels indices, nous avons combiné analyse multidimensionnelle et recherche de spécificités sur les trois corpus de notre étude : le péritexte des émissions, le péritexte des interactions et le texte des interactions.

\subsection{Indices génériques péritextuels : caractéristiques lexico- syntaxiques des présentations}

Selon notre modèle, les téléspectateurs des émissions de plateaux formulent immédiatement et automatiquement des hypothèses sur le ou les genres discursifs probablement en cours ou à venir dès le départ d'une émission (ou au moment de leur «arrivée » en cours d'émission). Parmi les différents «types » de paratextes (Genette, 1987), nous avons privilégié 
Intertexte générique et interprétation des actes de parole

l'étude du péritexte, au sein duquel nous avons analysé les éléments «formels » des présentations d'émissions et de leur "décor", les titres, les présentations des invités et les « annonces de genres ».

Dans la mesure où les émissions de plateaux voient se succéder différents genres d'interactions dont un ou deux dominants, nous avons mesuré les éventuelles correspondances entre caractéristiques lexico-syntaxiques des présentations et genre dominant de l'interaction. A cet égard, seuls quelques indices génériques peuvent être relevés dans les présentations des émissions. Au seuil de fréquence de 10, les deux premiers axes de l'AFC (croisant les 16 présentations d'émission avec leur vocabulaire) ont une valeur propre élevée $(20+13)$, mais ne permettent pas d'observer une proximité entre lexique des présentations et genre dominant. Pourtant, un premier indice est la «taille » moyenne des présentations des émissions (400 occurrences pour les émissions à dominante controverse contre 275 et 213 occurrences pour les émissions de jugement et de portrait). Parmi l'ensemble des lexèmes et syntagmes spécifiques des émissions de "controverse-polémique", « vous » $(+8)^{18}$ « vous avez » $(+4)$ « vous êtes » $(+4)$ et « il » (-5) indiquent une présentation " allocutive » caractéristique de ce type d'émission. Les émissions de jugement et de portrait sont à l'inverse caractérisées par une présentation " en absent » des invités (sous-utilisation des "vous » $(-6)$ et sur-emploi des « il » $(+3)$, « elle » $(+3)$ ou « c'est» $(+6)$ en portrait). Un autre indice des émissions de portrait à signaler est le mot «applaudissements» (+5). Ces spécificités se retrouvent au plan morphosyntaxique ${ }^{19}$ et sont complétées en particulier par

18. Les AFC et calculs de spécificités ont été réalisés à l'aide du logiciel Lexico 3 du Syled-Cla2t. Un coefficient de 8 indique que la probabilité d'un écart de répartition supérieur ou égal à celui que l'on a constaté était au départ de l'ordre de $10^{-8}$. Le signe (+ ou - ) indique un sur-emploi ou un sous-emploi dans la partie sélectionnée par rapport à l'ensemble du corpus. Nous avons utilisé les indices de spécificité pour les unités dont la fréquence est supérieure à 10, avec un seuil de probabilité fixé à 5\%.

19. Notre analyse morphosyntaxique s'est appuyée sur le logiciel Tropes et s’est limitée à la comparaison des fréquences de cinq méta-catégories 
Nicolas DESQUinabo

le sur-emploi des pronoms de première personne $(+3)$ et des modalisations $(+2)$ pour le portrait et le sous-emploi des modalisations de l'affirmation (-3) et de la cause (-3) pour le jugement.

\subsection{Autres types d'indices génériques péritextuels}

Parmi les nombreux autres types d'indices génériques péritextuels potentiels, le " décor » est un péritexte particulier dans la mesure où il est "parallèle " au texte et "continu ". Avec les signes vestimentaires, les signes du décor sont les éléments visuels les plus disponibles au cours d'une émission. Parmi l'ensemble des éléments potentiellement signifiants du décor (forme du plateau, couleurs, objets, etc.), nous avons systématiquement étudié les tailles du plateau et du public présent, le type de mobilier utilisé (chaises, canapés, tables, etc.), les orientations relatives des participants (qui fait face à qui ?) et le nombre d'animateurs et d'invités présents durant l'interaction. Cette brève analyse permet d'identifier quelques indices génériques potentiels :

- le genre portrait est généralement associé à la combinaison " grand plateau + public nombreux + fauteuil » (5/6 vs 3/18) et au nombre « restreint » d'invités (2 vs 4),

- les interactions de controverse et polémique se déroulent généralement dans les mêmes émissions caractérisées par un "petit plateau + public restreint» (8/12) et par un seul animateur (11/12 vs 2/12).

L'étroitesse de notre corpus d'émissions (16) nous invite bien sûr à ne pas extrapoler ces résultats. En revanche, la stabilité des éléments de décor permet à notre corpus de représenter une proportion importante (environ 30\%) des décors de la cinquantaine d'émissions régulièrement diffusées entre 2002 et 2004 sur les chaînes hertziennes françaises.

Les titres des émissions ne semblent pas présenter de corrélations lexico-syntaxiques avec le ou les genres à venir. En effet, les titres sont le plus souvent des syntagmes lexicalisant des thématiques générales (France, Europe, culture, destin,

(verbes, connecteurs, modalisations, adjectifs, pronoms) et de 38 souscatégories. 
images, actualité). Par ailleurs, le type d'identité des invités mis en avant dans les présentations est quasi-systématiquement le " statut professionnel » (journaliste, avocat, imitateur, chanteur, couturier, enseignante, ministre ou Miss France). Les invités de portrait sont le plus souvent des professionnels des arts et spectacles et les invités des émissions de controverse sont généralement des journalistes, des syndicalistes ou des politiques. Enfin, les genres à venir sont parfois explicités dans les présentations. Par exemple, dans Le fabuleux destin de..., Isabelle Giordano annonce : " on va voir aussi comment vous êtes devenu imitateur ». La visée de connaissance des étapes de la vie de l'invité, caractéristique du genre portrait, est ici clairement annoncée. Cependant, les genres sont généralement annoncés de manière moins explicite.

Ainsi, les indices péri-textuels lexicaux, syntaxiques ou sémantiques n'indiquent qu'une probabilité sur le genre dominant de l'émission. Par ailleurs, de nombreux téléspectateurs prennent ces émissions en cours et n'ont donc pas accès à ces indices. C'est pourquoi nous pensons que les interlocuteurs et surtout les téléspectateurs vont devoir confronter ces indices péritextuels à des indices textuels disponibles dans le déroulement même de l'interlocution.

\section{Controverse-Polémique}

« vous avez » $(+)$, « vous êtes » $(+)$, « il » (-), invités nombreux

+ petit plateau + public restreint

\section{Portrait}

« c'est » $(+)$, « il/elle » $(+)$, « applaudissements » $(+)$, grand plateau + public nombreux + fauteuil ou canapé

\section{Jugement}

« elle » (+), «vous » (-), «modalisations de la cause » (-) « modalisation de l'affirmation » (-), invités nombreux

Tableau n². Principaux indices génériques péritextuels

\subsection{Indices génériques lexico-syntaxiques textuels}

Afin d'étudier les éventuelles correspondances entre schémas pragmadiscursifs et configurations lexico-syntaxiques textuelles 


\section{NiCOLAS DESQUiNABO}

(vs péritextuelle), nous avons opéré une partition similaire de notre corpus d'interactions en distinguant les 24 «textes » des animateurs (4600 occurrences) des 24 "textes » des invités (11800 occurrences). Au seuil de fréquence de 10, les deux premiers axes de l'AFC (textes $\mathrm{x}$ lexique) des invités (graphique ${ }^{\circ} 1$ ) ont une valeur propre cumulée de $25(14+11)$. Comme le montre la comparaison des graphiques $n^{\circ} 1$ et $n^{\circ} 2^{20}$, le regroupement des invités du même genre supposé est plus ou moins net selon le plan d'analyse. Aux trois grands ensembles pragmadiscursifs (portrait, controverse et jugement-polémique) correspondent deux grands ensembles lexicaux (portraitjugement et controverse-polémique) ${ }^{21}$. En revanche, au moins cinq des six interactions de chaque genre sont regroupées dans les deux analyses.

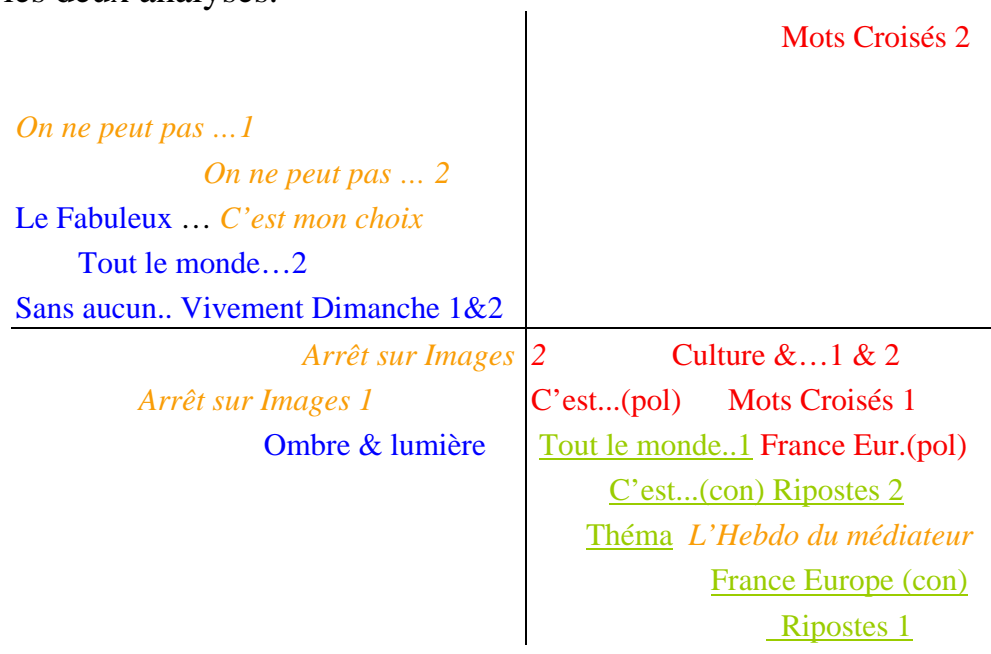

Graphique ${ }^{\circ} 1$ : Premier plan de l’AFC lexicale des invités ${ }^{22}$

20. Le graphique $n^{\circ} 2$ correspond à l'ACP évoquée au §2.2 et étudiée dans Desquinabo (2005).

21. Les proximités suggérées par les deux premiers facteurs de l’AFC lexicale des invités sont quasi-identiques à celles des plans suivants et celles issues de l'analyse « hors individu atypique » (Mots Croisés 2).

22. Dans les deux graphiques, les interactions de portrait sont en bleu, les interactions de controverse en vert, les interactions de polémique en rouge et les interactions de jugement en orange. 
Intertexte générique et interprétation des actes de parole

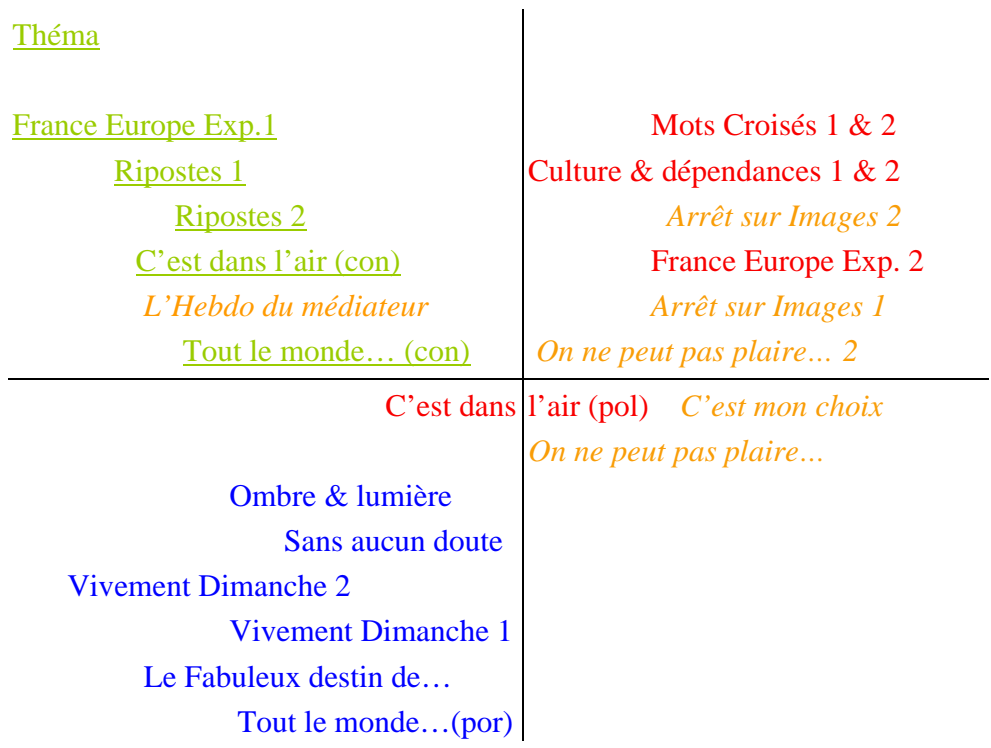

Graphique n² : Premier plan de l’ACP « pragma-discursive » des invités ${ }^{23}$

Il est intéressant d'observer qu'aux deux plans de l'analyse, L'Hebdo du médiateur, initialement catégorisée dans le genre jugement, s'est déroulée comme une interaction typique de controverse $^{24}$. Le principal résultat non attendu de l'AFC lexicale des invités est la forte proximité entre trois jugements et le groupe de portraits.

Contrairement au cas des invités, l'AFC lexicale des animateurs distingue peu les interactions et encore moins les genres. Ce résultat rejoint en partie celui de l'ACP pragmadiscursive qui n'a permis d'observer que des regroupements génériques partiels. La proportion importante des actes de gestion (présentations, proposition de thèmes, etc.) dans les textes parfois très courts des animateurs explique probablement

23. L'axe horizontal est principalement corrélé à la proportion d'actes de blâmes et aux tours de parole courts $(\mathrm{VP}=28 \%)$ et l'axe vertical à la proportion d'actes d'information ( $\mathrm{VP}=16 \%$ ).

24. Voir notre analyse détaillée de ce «détournement " générique dans Desquinabo (2007). 
la relative faiblesse des spécificités génériques des animateurs par rapport aux nombreuses spécificités des invités (cf. tableau $\mathrm{n}^{\circ} 3^{25}$ ).

\begin{tabular}{|c|}
\hline Cont \\
\hline $\begin{array}{l}\text { Animateur = « ce que » (+3), « com } \\
\text { Invités = «de l’ " (+5), " qu’il } \\
\text { "nous » (+3), " était » (-5), " ve } \\
\text { /manière/ (+3), /première personne/ }\end{array}$ \\
\hline \\
\hline $\begin{array}{l}\text { las » }(-3) \\
\text { lis » (+6), « le / l' } \\
\text { /affirmation/ (+2), } \\
\text { c. }\end{array}$ \\
\hline \\
\hline 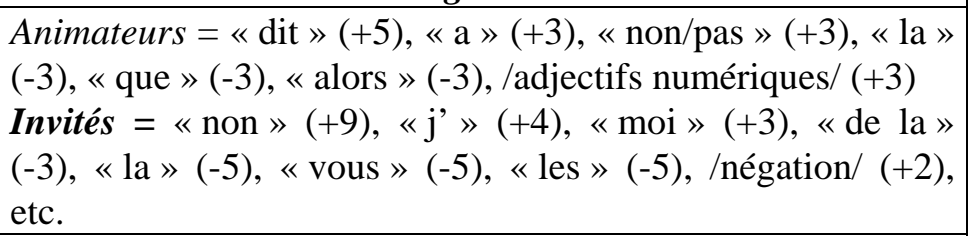 \\
\hline \\
\hline $\begin{array}{l}\text { Animateur = « alors » }(+7), \\
\text { Invités = « vous » }(+22), \\
(-4), \text { « euh » }(-5), \text { « elle » }\end{array}$ \\
\hline
\end{tabular}

Tableau n³. Indices génériques lexico-syntaxiques textuels

Ainsi, malgré la taille restreinte de notre corpus, il est possible d'identifier de multiples indices génériques lexicaux et morphosyntaxiques. La combinaison de ceux-ci aux indices pragmadiscursifs permet de distinguer et identifier les quatre genres d'activités discursives de notre étude. Cependant, à la différence des indices «formels » péritextuels et des indices

25. A l'aide du retour au contexte, nous avons exclu les spécificités concentrées dans un ou deux textes (par exemple, le lexème " affaire " est en apparence spécifique du genre jugement $(+7)$, alors que ses occurrences sont concentrées dans deux interactions). 
Intertexte générique et interprétation des actes de parole

morphosyntaxiques et lexicaux, la caractérisation des indices pragmadiscursifs nécessite au préalable l'interprétation des différents actes de parole se succédant au cours des textes. Ces indices permettant en retour de formuler des hypothèses sur l'activité en cours, contribuant ainsi à orienter l'interprétation des actes de parole. Voyons comment notre modélisation précise les parcours possibles de ce « cercle herméneutique » au palier de la période.

\section{Exemples d'actualisations d'actes de parole}

Trois exemples nous permettront d'illustrer nos hypothèses sur l'interprétation des actes de parole d'un texte interlocutif. Ces exemples simuleront une interprétation au fil du texte et illustreront également une particularité des corpus d'interactions : la possibilité d'accéder aux interprétations « en acte » manifestées par les interlocuteurs (Gumperz, 1989 : 56). Ces interprétations (plus ou moins manifestées) sont à la fois une source d'observation de certains parcours interprétatifs et une des composantes orientant le parcours interprétatif que nous tenterons de décrire ici : celui de l' « analyste-téléspectateur ${ }^{26}$ que nous sommes.

\subsection{Premiers actes d'une interaction de jugement}

Les divers indices formels disponibles dans la présentation de l'émission On ne peut plaire à tout le monde (applaudissements $(+)$, elle $(+)$, vous $(-)$, « grand plateau + public nombreux + deux animateurs ») annoncent la venue probable d'interactions des genres jugement ou portrait. L'interprétation de la présentation de deux des trois invités présents dans l'interaction analysée (Miss France et Mme de Fontenay) soutient plutôt l'hypothèse d'une activité de jugement : après une information et deux citations sur le fait que ces invitées se sont crêpées le chignon, la co-animatrice pose la question-thème (car manifestement non adressée) : quinze jours après sont-elles les

26. Nous avons généralement interprété la valeur illocutoire "mutuellement manifeste » (Chabrol \& Bromberg, 1999 : 293) même si celle-ci n’est pas toujours disponible (Kerbrat-Orecchioni, 2005: 79-81) pour différentes raisons (politesse, changements de thème, etc.). 
meilleurs amies du monde? et annonce: c'est ce que nous verrons tout à l'heure. En revanche, une éventuelle confrontation entre Mme de Fontenay et Guy Bedos n'est pas annoncée dans la présentation. Pourtant, après de longues salutations, l'animateur Marc-Olivier Fogiel demande :

- Fogiel : «Est-ce que l’institution Guy Bedos aime bien euh l'institution Madame de Fontenay ?»

Cet acte peut être interprété comme une «demande de compliment » annonçant la venue d'une interaction de portrait ou comme une "demande de blâme " annonçant plutôt une interaction de jugement. La modalité « demande » est à la fois indiquée par des indices linguistiques (est-ce que + prosodie interrogative) et par la fréquence de cette modalité dans le rôle d'animation en général. La classe d'acte demandée («relationnel») est indiquée par la présence des deux protagonistes de la proposition sur le plateau et par le verbe aimer. En revanche, le choix entre les valeurs de compliment ou de blâme doit attendre l'intervention suivante :

- Bedos : « ah, ça commence très très dur ! la vache ! »

Cette intervention semble débuter par un jugement «métacommunicationnel » sur l'interaction (ça) à connotation négative (dur) et enchaîne sur un acte court qui peut avoir valeur d'appréciation ou de blâme de l'animateur. Ce refus de répondre (temporaire) ne permet pas d'interpréter selon le " principe séquentiel » (Schegloff \& Sacks, 1973). L’hypothèse d'un refus de répondre est avant tout fondée sur la combinaison d'indices linguistiques aux normes du genre jugement dans lequel les refus de répondre et contestations métacommunicationnelles sont fréquents. Simultanément, l'intervention de Bedos semble manifester qu'il a interprété le premier acte comme une demande de blâmer, ce qui renforce l'interprétation de ce début d'interaction comme une activité de jugement débutée localement par une demande de blâme. Ces interprétations et la valeur de blâme de la vache sont confirmées par l'intervention suivante de l'animateur qui «reconnaît » le blâme de l'invité tout en indiquant que sa première intervention était bien une demande de blâme : 
Intertexte générique et interprétation des actes de parole

- Fogiel : « (rires) quel salaud ce Fogiel !»

La suite de l'interaction verra se prolonger l'activité de jugement, assez nettement indiquée par une combinaison de la plupart des indices du genre : tours très courts chevauchés, blâmes et moqueries de l'animateur, démentis et moqueries des invités, applaudissements réguliers, sur-emploi de la première personne et des négatifs, sous-emploi des articles et noms, etc.

\subsection{Liens entre les actes d'une interaction de controverse}

Aucun indice situé dans la présentation de l'émission ou le décor ne semble annoncer la controverse de Tout le monde en parle entre Thierry Ardisson et Alain Madelin. La présentation de l'invité en début d'interaction peut autant être interprétée comme le début d'une interaction de controverse : député UMP (...) vous êtes favorable à la guerre en Irak ou de polémique: est-ce que c'est pas une posture pour vous singulariser? Le déroulement du début de l'interaction valide plutôt la première hypothèse (tours de parole long, absence de blâmes et de vous, etc.). Après une minute et au septième tour de parole, l'animateur Ardisson cite son invité Madelin (indice linguistique : vous dites) :

- Ardisson : «Alors vous dites « la position qui consisterait le cas échéant à se passer de l’ONU serait légitime, notre place est au côté de l'Amérique »

Nous situant dans une interaction de controverse, cette citation a probablement plus une valeur illocutoire de «demande de justification d'une évaluation » qu'une valeur d' «accusation » ou de « demande de confirmation ». Cette interprétation semble confirmée par la longue réponse de l’invité :

- Madelin : «Les dix-sept résolutions de l'ONU / ${ }^{27}$ qui n’ont pas été respectées par Saddam Hussein / donnent le droit, aux Etats membres d'intervenir le cas échéant pour faire respecter ce droit »

27. Les « / » délimitent les actes de parole successifs. 
contient un acte d'information qui argumente en faveur de l'acte « englobant » légitimant l'intervention des Etats membres et validant ainsi la citation de l'animateur ${ }^{28}$.

Le troisième acte de la réponse de Madelin : ce n'est pas une dictature comme toutes les autres est " linguistiquement » une comparaison. Son insertion dans une controverse rend très probable sa valeur interactive de justification de l'acte précédent ou suivant :

- « parce que lui il a perdu une guerre / il en est résulté des obligations internationales / deux obligations internationales / la première, respecter les droits humains sur le sol Irakien / ce n’est pas respecté / la seconde, désarmer / ce n'est pas respecté ».

Ce macro-acte se présente comme une explication argumentant en faveur (parce que) du troisième acte à l'aide de sept actes d'information. Les deux premiers présentent un lien de succession, le troisième explicite le second et est explicité par les quatrième et sixième actes. Et les deux ce n'est pas respecté se présentent comme des informations venant argumenter en faveur du troisième acte ce n'est pas un dictateur comme les autres. Globalement, ce macro-acte argumente en faveur de ce n'est pas un dictateur comme les autres à partir d'informations, et il rétro-interprète également l'acte ce n'est pas un dictateur comme les autres en "justification » supplémentaire de l'acte légitimant l'intervention sans l'ONU, confirmant ainsi les attentes interprétatives. Ce type de rétro-interprétation à " courte distance " est très fréquent dans notre corpus, contrairement aux rétro-interprétations «tardives » (après plusieurs échanges) qui sont plus rares mais modifient parfois nos hypothèses sur le genre d'activité en cours ${ }^{29}$. Ces négociations du genre sont particulièrement fréquentes dans les

28. La valeur de "validation » peut être interprétée à partir de connaissances du domaine s'agissant de l'équivalence entre « se passer de l'ONU » et «donner le droit aux Etats membres d'intervenir » et/ou en se fondant sur l'absence d'indices linguistiques de rectification de la citation qui indiquent sa validation de facto.

29. Voir Desquinabo (2005 : 96). 
Intertexte générique et interprétation des actes de parole

émissions au cours desquelles controverses et polémiques se succèdent.

\subsection{Négociation d'un changement d'activité discursive}

La présentation et le décor de l'émission C'est dans l'air sont assez typiques des émissions présentant principalement des controverses et accessoirement des polémiques $^{30}$ : la longue présentation des quatre invités multiplie les vous avez et vous êtes, alors qu'aucun il n'est prononcé. De plus, le plateau est petit et le public restreint. La demande de validation de l'animateur (c'est une dictature qui profite (de la guerre en Irak) pour régler des comptes) est validée et longuement argumentée par le premier invité puis invalidée par l'invitée Janette Habel (je dirais pas ça du tout), ce qui est typique du genre controverse, comme les fréquents adverbes de manière, les nombreux il y a et qu'il ou la quasi-absence de vous. Arrive alors une nouvelle "demande de validation" assez explicite adressée par l'animateur Yves Calvi au troisième invité Jacobo Machover :

- Calvi : « Jacobo Machover, qu'est ce que vous pensez de cette interprétation de ce qui se passe dans votre pays en ce moment? »

Le premier acte de la réponse de l'invité va alors proposer un changement d'action: la controverse s'interrompt et la polémique (entre Machover et Habel) est déclenchée :

- Machover : «Il me semble que c'est tout simplement de la propagande Castriste »

Cet acte nous semble être une «accusation» directe, essentiellement en raison de la connotation négative du terme propagande, notamment lorsqu'il est qualifié de castriste par un invité présenté comme anti-castriste. Cette interprétation reste une hypothèse, mais celle-ci est confirmée par la réaction de l'« accusée » qui interrompt l'argumentation de l'« accusateur » :

30. C'est dans l'air présente également beaucoup d'interactions du genre expertise qui n'a pas été analysé dans cette recherche. Pour plus de détails sur cette émission et ce genre, voir Desquinabo (2005 : 117-118 et 195-196). 


\section{NiCOLAS DESQUiNABO}

- Machover : « les américains n’ont à aucun moment menacé Cuba depuis fort longtemps d'une intervention dans ce pays / Le castrisme, le »

- Habel : « C’est absolument faux hein !»

- Machover : « vous permettez je vous ai laissé parler»

- Habel : « Oui, mais c’est faux »

Plusieurs indices très typiques du genre polémique sont combinés dans les échanges qui suivent l'acte hypothétique d'accusation: la durée des tours de parole est fortement raccourcie, les interruptions et chevauchements se multiplient, les je et les vous apparaissent et Habel « infirme » explicitement les propos de Machover (c'est faux) qui lui « conteste son tour de parole ». Après cet échange, Machover reformulera son accusation, ce qui achèvera de confirmer la valeur d' « accusation » du premier acte, pourtant peu attendu dans une controverse. L'énonciation de Il me semble que c'est tout simplement de la propagande Castriste réalise donc à la fois un acte d'accusation et un acte modifiant le contexte d'interprétation (Filliettaz, 2006) car proposant une nouvelle activité : la polémique. Cette nouvelle activité sera ratifiée par l'«accusée » (ce qui est fréquent), mais sera rapidement interrompue par l'animateur (ce qui est plus rare). En effet, l'intervention de l'animateur (attendez, vous, vous lui répondrez plus tard) ajournera temporairement cette polémique qui reprendra vingt minutes plus tard durant quelques minutes avant d'être à nouveau interrompue par l'animateur. Ainsi, la seule prise en compte du genre d'activité en cours n'aurait pas permis d'attribuer la valeur d' "accusation" à l'acte initial de Machover. Des hypothèses sur les connotations négatives d'un syntagme (propogande castriste) et la comparaison des caractéristiques des réactions suivant son énonciation à l'intertexte des émissions de plateaux nous ont permis d'identifier un changement de genre et de valider a posteriori l'hypothèse interprétative initialement formulée.

\section{Conclusion}

L'approfondissement des connaissances sur le rapport entre paliers contextuels et processus interprétatifs doit selon nous 
Intertexte générique et interprétation des actes de parole

surmonter au moins deux difficultés majeures. Tout d'abord, l'étude des interactions entre les différents types d'indices génériques sur des corpus davantage représentatifs de l'intertexte d'un champ générique donné est limitée par l'impossibilité d'une analyse automatique des actes de parole. A ce problème essentiellement économique s'ajoute le problème plus complexe posé par le repérage des changements d'activités discursives dans les «discours-en-interaction » (KerbratOrecchioni, 2005 : 74-78 ; Filliettaz, 2006). Quelques pistes ont été explorées, mais la délimitation de textes génériquement " homogènes » dépasse la simple interrogation sur le « mélange des genres » à la télévision. La plupart des rencontres (réunions de travail, repas entre amis, etc.) voient se succéder et s'enchâsser des genres discursifs très variés dont les limites se trouvent être très rarement marquées "formellement". Ce problème est selon nous fondamental pour la linguistique de corpus en général et pour le repérage automatique des genres en particulier (Malrieu \& Rastier, 2001). En effet, les récurrences et spécificités de certains genres peuvent être sous-estimées par la sélection de corpus génériquement hétérogènes. Et les spécificités discursives de certaines organisations, groupes ou individus risquent d'être sur-estimées par la sélection de corpus différemment pondérés du point de vue des genres discursifs utilisés.

Par ailleurs, si les interactions entre contexte générique et interprétation des actes de parole sont selon nous primordiales, notre modélisation doit s'enrichir des autres types d'indices génériques que sont probablement les récurrences thématiques $^{31}$, les types de progression topicale ou l'organisation des énonciateurs et points de vue (Rabatel, 2005). L'intégration de ces dimensions du discours dans notre modélisation permettrait à la fois d'affiner la description des genres de discours et de rendre compte de manière moins imparfaite des processus d'interprétation des actes de parole.

31. Par exemple, dans les interactions de portrait, le thème de la famille semble très récurrent à la fois par rapport aux autres thèmes et par rapport à la place de ce thème dans les autres genres. 
Nicolas DESQUinabo

\section{Références bibliographiques}

Adam J.-M. \& Heidmann U. (2004). « Des genres à la généricité. L'exemple des contes (Perrault et Grimm) », Langages $153: 62-72$.

Bange P. (1989). «Analyse conversationnelle et théorie psychologique de l'action », Verbum 12 : 27-41.

Bouchard R. (1988). "La conversation-palimpseste ", in J. Cosnier, N. Gelas \& C. Kerbrat-Orecchioni (éds) Echanges sur la conversation. Paris : CNRS, pp. 105-121

Bronckart J.P (1997). Activité langagière, textes et discours. Lausanne : Delachaux et Niestlé.

Burger M. (2001). "La dimension interactionnelle», in E. Roulet et al. (éds) Un modèle et un instrument d'analyse de l'organisation des discours. Berne : Lang, pp. 139-164.

Chabrol C. \& Bromberg M. (1999). " Préalables à une classification des actes de parole ", Psychologie française 44, 4 : 291-306.

Charaudeau P. (1995). "Le dialogue dans un modèle de discours », Cahiers de Linguistique Française 17: 141178.

Coirier P., Gaonac'h D. \& Passerault J.M (1996). Psycholinguistique textuelle. Paris : A.Colin / Masson.

Desquinabo N. (2005). Caractéristiques et contraintes des genres interlocutifs dans les émissions de plateaux télévisées. Thèse de doctorat. Université Paris 3.

Desquinabo N. (2007). « Analyse des co-constructions de rôles discursifs émergents dans les émissions de plateaux télévisées », Revue Internationale de Psychosociologie 29 : 91-108.

Filliettaz L. (2001). « La dimension référentielle », in E. Roulet et al. (éds) Un modèle et un instrument d'analyse de l’organisation des discours. Berne : Lang, pp. 97-137.

Filliettaz L. (2006). «La place du contexte dans une approche praxéologique du discours. Le cas de l'argumentation dans les interactions scolaires », Pratiques 129-130 : 71-88. 
Intertexte générique et interprétation des actes de parole

Genette G. (1979). Introduction à l'architexte. Paris : Seuil.

Genette G. (1987). Seuils. Paris : Seuil.

Gumperz J.J. (1989). Engager la conversation. Introduction à la sociolinguistique interactionnelle. Paris : Minuit.

Hutchby I. \& Wooffitt R. (1998). Conversation Analysis. Cambridge : Polity Press.

Hymes D. (1980). « Modèles pour l'interaction du langage et de la vie sociale », Etudes de linguistique appliquée 37 : 127153.

Kerbrat-Orecchioni C. (2000). «Quelle place pour l'émotion dans la linguistique du XXème siècle? Remarques et aperçus », in C. Plantin, M. Doury \& V. Traverso (éds) Les émotions dans les interactions. Lyon : PUL, pp. 33-74.

Kerbrat-Orecchioni C. \& Traverso V. (2004). «Types d'interactions et genres de l'oral », Langages $153: 41-51$.

Kleiber G., Adam J.M., Combettes B., Maingueneau D. \& Moirand S. (2006). "Introduction au(x) co(n)texte(s) ", Pratiques 129-130 : 17-49.

Lebart L. \& Salem A. (1994). Statistique Textuelle. Paris : Dunod.

Malrieu D. \& Rastier F. (2001). "Genres et variations morphosyntaxique », Traitement Automatique des langues 42, 2 : 548-577. http://www.revue-texto.net

Rabatel A. (2005). «La part de l'énonciateur dans la construction interactionnelle des points de vue », Marges linguistiques $9: 136$. http://www.marges-linguistiques.com

Rastier F. (1998). " Le problème épistémologique du contexte et le statut de l'interprétation dans les sciences du langage », Langages 129 : 97-111.

Rastier F. (2001). Arts et sciences du texte. Paris : PUF.

Rastier F. et al. (éds) (1994). Sémantique pour l'analyse : de la linguistique à l'informatique. Paris : Masson.

Roulet E. et al. (éds) (2001). Un modèle et un instrument d'analyse et d'organisation du discours. Berne : Lang. 
Nicolas Desquinabo

Schegloff E.A. (1984). " On some questions and ambiguities in conversation ", in J.M Atkinson \& J. Heritage (eds) Structures of Social Action. Studies in Conversation Analysis. Cambridge : Cambridge University Press, pp. 2852.

Schegloff E.A. \& Sacks H. (1973). «Opening up closings », Semiotica 7 : 289-327.

Searle J.R. (1982). Sens et expression. Paris: Editions de Minuit.

Vernant D. (1997). Du discours à l'action. Paris : PUF. 